\title{
Welcome to Journal of Hepatocellular Carcinoma
}

\author{
This article was published in the following Dove Press journal: \\ Journal of Hepatocellular Carcinoma \\ 9 January 2015 \\ Number of times this article has been viewed
}

\section{Ahmed O Kaseb \\ Department of Gastrointestinal Medical Oncology, The University of Texas MD Anderson Cancer Center, Houston, TX, USA}

Correspondence: Ahmed O Kaseb Department of Gastrointestinal Medical Oncology, Unit 426, The University of Texas MD Anderson Cancer Center, I5I5 Holcombe Boulevard, Houston, TX 77030, USA

Tel +17137922828

$\mathrm{Fax}+17 \mid 3745$ II63

Email akaseb@mdanderson.org
Hepatocellular carcinoma (HCC) develops as a consequence of underlying chronic liver disease, most commonly cirrhosis. Therefore, HCC management draws on the expertise of a wide range of medical specialists. Currently, novel therapeutic modalities for HCC are investigated within the framework of a multidisciplinary approach. Therefore, there is a critical need for a dedicated journal for HCC with special emphasis on understanding pathogenesis, emerging diagnosis and treatment approaches, and translational research strategies.

The new journal Journal of Hepatocellular Carcinoma strives to be the essential clinical guide for medical and surgical oncologists, hepatologists, interventional radiologists, and all physicians and researchers involved in the care of patients with HCC. Our journal is committed to building an intercontinental community of researchers and clinicians to encourage establishment of collaborations and development of improved strategies to advance the field and help our patients from a global prospective. $\mathrm{HCC}$ is a very heterogeneous disease with multiple risk factors and noted differences in natural history and treatment outcomes based on demographics, risk factors, and even geographic locations, as evident by the noted difference in the magnitude of effects and rate of adverse events based on recent clinical trials conducted on different continents using the same systemic or local therapy modality. ${ }^{1}$ Therefore, one of the ultimate goals of the journal is to advance our understanding of the biology and natural history of HCC given the differential outcome based on these variables.

Journal of Hepatocellular Carcinoma is an international, peer-reviewed, openaccess journal encompassing all aspects of HCC. The journal seeks to publish outstanding research articles, reviews, mini-reviews, perspectives, commentaries and controversies, and editorials in all major areas of HCC. We believe that a substantial knowledge that can be derived from negative HCC studies remains unavailable, most likely because of under-reporting. Therefore, we accept negative HCC studies submissions. We also encourage unsolicited reviews and welcome critical analysis of published manuscripts in the form of letters to the editor and commentaries. Our team recognizes that knowledge related to $\mathrm{HCC}$ is dispersed across many clinical and academic disciplines. Therefore, our mission is to bridge this gap between researchers and clinicians to provide interdisciplinary publications and promote communication among different specialties. The scope of our journal includes broad basic, translational, and clinical topics such as (but not limited to) epidemiology, hepatocarcinogenesis, diagnosis, staging, radiogenomics, cancer biomarkers, systemic therapy, radiation, 
surgery, translational oncology, multidisciplinary management, and cost effectiveness.

Given the fast-emerging field of HCC biomarkers for early diagnosis, prognosis, and therapeutic targeting, it is critical to report comprehensive and detailed information related to all steps involved in the research process, including study design, sample collection, processing, and methodology of biomarker assay and statistical analysis. This approach will facilitate interpretation of biomarker study results and comparison of results from different studies, in addition to improving the chances of reproducing the biomarkers results in future validation studies. This is imperative to the success of biomarker research in $\mathrm{HCC}$, which is expected to advance the field through discovery and validation of biomarkers related to early diagnosis, risk assessment, prognosis, prediction of response or resistance to therapy, and identification of new therapeutic targets. Therefore, Journal of Hepatocellular Carcinoma will assign highest priority to biomarker papers that are REMARK (reporting of tumor marker studies) compliant. REMARK guidelines have been established by the National Cancer Institute-European Organization for Research and Treatment of Cancer working group. ${ }^{2}$ While the guidelines in their original form were intended for use in prognostic biomarker studies, we encourage authors of other biomarker studies, such as biomarkers of early diagnosis or prediction of therapy outcome, to adhere to REMARK guidelines as applicable to their studies. Similarly, Journal of Hepatocellular Carcinoma will assign priority to papers that are compliant with emerging reporting guidelines, such as CONSORT Statement for reporting randomized trials ${ }^{3}$, and STROBE Statement for reporting observational studies in epidemiology $y^{4}$, in an effort to reduce the influence of poor or biased reporting.

We believe that the peer review process has a very valuable role in the journal's editorial process. Nevertheless, we acknowledge that peer review is imperfect. Therefore, to augment the peer-review processes, manuscripts submitted to Journal of Hepatocellular Carcinoma will generally be reviewed by at least three experts with the aim of reaching the first decision within 14 days.

In conclusion, our goal is to integrate knowledge across multiple disciplines to achieve new insights in HCC and to provide a landscape for researchers and clinicians to focus on emerging topics in HCC though this first dedicated journal for HCC.

\section{Disclosure}

The author reports no conflict of interest in this work.

\section{References}

1. Kaseb AO. Beyond the basics: the differential effects of demographics and hepatitis status on treatment outcome in hepatocellular carcinoma. Oncology. 2013;85(1):41-43.

2. McShane LM, Altman DG, Sauerbrei W, et al. Reporting recommendations for tumor marker prognostic studies. J Clin Oncol. 2005;23(36):9067-9072.

3. Moher D, Schulz KF, Altman D, Group C. The CONSORT statement: revised recommendations for improving the quality of reports of parallelgroup randomized trials. JAMA. 2001;285(15):1987-1991.

4. von Elm E, Altman DG, Egger M, et al. The Strengthening the Reporting of Observational Studies in Epidemiology (STROBE) statement: guidelines for reporting observational studies. PLoS medicine. 2007; 4(10):e296.
Journal of Hepatocellular Carcinoma

\section{Publish your work in this journal}

The Journal of Hepatocellular Carcinoma is an international, peerreviewed, open access journal that offers a platform for the dissemination and study of clinical, translational and basic research findings in this rapidly developing field. Development in areas including, but not limited to, epidemiology, vaccination, hepatitis therapy, pathology and

\section{Dovepress}

molecular tumor classification and prognostication are all considered for publication. The manuscript management system is completely online and includes a very quick and fair peer-review system, which is all easy to use. Visit http://www.dovepress.com/testimonialsphp to read real quotes from published authors. 